\title{
PIV analysis of Merging Flow in a Simplified Model of a Rotary Kiln
}

\author{
I. A. S. Larsson*, B. R. Granström*, T. S. Lundström* and \\ B. D. Marjavaara ${ }^{\dagger}$
}

Rotary kilns are used in a variety of industrial applications. The focus in this work is on characterizing the non-reacting, iso-thermal flow field in a rotary kiln used for iron ore pelletization. A downscaled, simplified model of the kiln is experimentally investigated using Particle Image Velocimetry. Five different momentum flux ratios of the two inlet ducts to the kiln are investigated in order to evaluate its effect on the flow field in general and the recirculation zone in particular. Time-averaged and phase-averaged analyses are reported, and it is found that the flow field resembles that of two parallel merging jets, with the same characteristic flow zones, as exemplified in Fig. 1. The back plate separating the inlet ducts acts as a bluff body to the flow and creates a region of reversed flow behind it. Due to the semi-circular cross-section of the jets, the wake is elongated along the walls. Conclusions are that the flow field shows a dependence on momentum flux ratio of the jets; as the momentum flux ratio approaches unity, there is an increasing presence of von Kármán-type coherent structures with a Strouhal number of between 0.16-0.18. These large-scale structures enhance the mixing of the jets and also affect the size of the recirculation zone. Due to the geometrical confinement the flow field in the kiln deviates from the flow field behind a bluff body in a free stream. The vortical structures converge towards the kiln walls, leading to a coupling between the wall shear layer and the vortex originating from the shear layer of opposite vorticity. This interaction results in a portion of the wall shear layer being ejected by the influence of the shed vortices.

*Division of Fluid and Experimental Mechanics, Luleå University of Technology, SE-97187 Luleå, Sweden

${ }^{\dagger}$ LKAB, Kiruna, Sweden

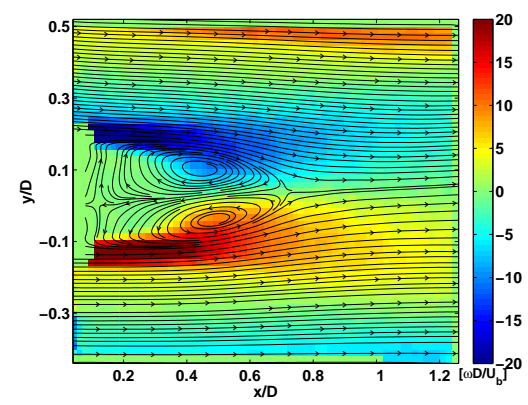

(a) Time-average

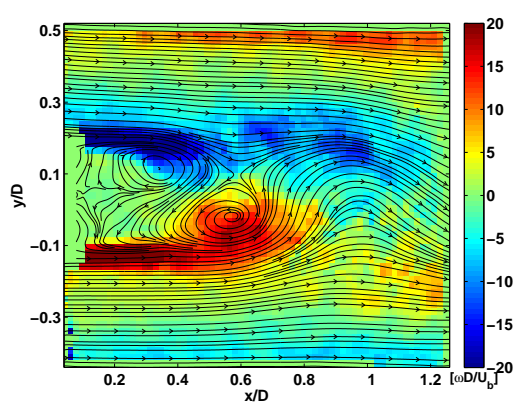

(b) Phase-average

Figure 1: Time-averaged and phase-averaged results for matched momentum flux. The contours are coloured by vorticity and streamlines are added to highlight the flow patterns. 